\title{
Linx
}

Revue des linguistes de l'université Paris X Nanterre

48 | 2003

Approches syntaxiques contemporaines

\section{La Role and Reference Grammar, une grammaire de l'interface entre syntaxe, sémantique et pragmatique}

Jacques François

\section{(2) OpenEdition \\ Journals}

Édition électronique

URL : http://journals.openedition.org/linx/208

DOI : $10.4000 / \operatorname{lin} \times .208$

ISSN : 2118-9692

Éditeur

Presses universitaires de Paris Nanterre

\section{Édition imprimée}

Date de publication : 1 juin 2003

Pagination : 77-89

ISBN : 0246-8743

ISSN : 0246-8743

Référence électronique

Jacques François, " La Role and Reference Grammar, une grammaire de l'interface entre syntaxe,

sémantique et pragmatique », Linx [En ligne], 48 | 2003, mis en ligne le 01 octobre 2003, consulté le 06 mai 2019. URL : http://journals.openedition.org/linx/208 ; DOI : 10.4000/linx.208 


\title{
La Role and Reference Grammar, une grammaire de l'interface entre syntaxe, sémantique et pragmatique
}

\author{
Jacques François, Université de Caen E CRISCO, UMR 6170 \\ jacques.francois@crisco.unicaen.fr
}

Le titre de cet article est directement inspiré de celui de l'ouvrage en voie de parution de Robert D. van Valin Jr., The Syntax-Semantics-Pragmatics Interface: an Introduction to Role and Reference Grammar. Goldsmith \& Huck (1991) classent les théories grammaticales dans deux types, les grammaires de DISTRIBUTION, centrées sur la bonne formation des combinaisons de constituants morphosyntaxiques, et celles de MÉDIATION, centrées sur la corrélation entre structure morphosyntaxique et structure (pragma-)sémantique. Dans le premier chapitre de leur somme consacrée à la Role and Reference Grammar ${ }^{1}$ — désormais «RRG» - van Valin \& LaPolla (1997) développent ce classement et montrent que le type 'corrélatif' auquel ils rattachent la RRG compte une plus grande variété de modèles (par exemple la Grammaire Fonctionnelle de S.C. Dik, la Grammaire Lexico-Fonctionnelle de J. Bresnan, la Grammaire de Construction de Ch. Fillmore, P. Kay et A. Goldberg, la Grammaire HPSG, la Grammaire Cognitive de R. Langacker, la Grammaire Sens-Texte d'I. Melçuk, etc.) que le type qu'ils désignent comme «centré sur la syntaxe»,

\footnotetext{
1 On pourrait traduire littéralement l'intitulé de cette théorie : Grammaire des Rôles et de la Référence. Le principe fondateur du modèle, qui lui donne son nom, est que le caractère universel dominant des langues du monde est la présence dans tout énoncé linguistique de constituants à fonction prédicative et de constituants à fonction référentielle, quel que soit le mode de réalisation morphosyntaxique particulier de ceux-ci. Dans un énoncé, tout constituant référentiel contribue à l'exercice de la fonction d'un constituant prédicatif par l'intermédiaire d'un rôle sémantique. Les rôles ne sont pas énumérés, mais sont des abréviations de fonctions hiérarchisées dans l'entrée lexicale du constituant prédicatif, laquelle constitue le Cœur (dans un sens technique défini plus bas) de la Structure Logique.
} 
Jacques François

principalement représenté par les modèles développés successivement par N. Chomsky².

\section{Une grammaire dédiée à l'interface entre quatre composantes}

Mon propos sera ici exclusivement d'illustrer aussi clairement que possible ce que Robert van Valin entend concrètement par « interface entre syntaxe, sémantique et pragmatique », c'est-à-dire comment coopèrent les quatre composantes de la RRG, la Structure logique, la Projection des constituants, la Projection des opérateurs et la Projection pragmatique ${ }^{3}$. On trouvera d'autres développements sur le traitement par la $R R G$ de l'influence des propriétés de quantification véhiculées par la projection des opérateurs du syntagme nominal objet direct sur les propriétés de caractère aspectuel de la projection du prédicat verbal dans François (à paraître 2) et sur le traitement de la syntaxe et de la morphologie dérivationnelle du verbe français dans le comparatif de la RRG et de la Grammaire Fonctionnelle de Simon Dik $(1989,1997)$ dans François (à paraître 1).

La Role and Reference Grammar distingue quatre composantes :

- la structure logique qui fournit une représentation logico-sémantique de l'énoncé dans un format dérivé de Dowty (1979), sans reprendre cependant l'appareil proprement logique de la 'théorie des modèles' que D. Dowty emprunte à R. Montague (quantification sur les individus et les événements, lambda-abstraction),

- la projection des constituants, qui constitue un mode de représentation syntaxique constitué de 'pièces syntaxiques' (templates) à fonction soit prédicative soit référentielle ${ }^{4}$ (cf. section III ci-dessous),

- la projection des opérateurs, disposée en miroir de la projection des constituants, qui traite les catégories fonctionnelles (aspect, temps, modalité, force illocutoire) de la structure stratifiée de la proposition (exerçant une fonction prédicative) et celles (détermination déictique et anaphorique, quantification, qualification) de la structure stratifiée du SN (chargé de l'exercice de la fonction référentielle),

- et enfin la projection pragmatique qui contribue avec la structure logique à sélectionner les pièces syntaxiques de la projection des constituants en pointant sur les constituants marqués comme exerçant une fonction topicale ou focale ${ }^{5}$.

2 Voir la présentation comparative des théories de Grammaire Relationnelle, Grammaire LexicoFonctionnelle, Principes \& Paramètres, Grammaire HPSG et RRG qui constitue le chapitre 5 de van Valin (2001).

3 La notion de 'projection' ne s'entend pas comme dans la syntaxe 'X-barre' : il ne s'agit pas de la couverture de têtes syntaxiques de différente extension $(\mathrm{X}, \mathrm{X}$ ', $\mathrm{X}$ " : projection maximale), mais de trois points de vue complémentaires sur la structuration de l'énoncé. La Projection des constituants et celle des opérateurs associent un matériau lexical et morphologique à des segments de la Structure logique. En revanche la Projection pragmatique fournit des données de structure informationnelle absentes de la Structure logique. La justification du terme 'projection' est donc discutable dans ce dernier cas.

${ }^{4}$ Sur les propriétés originales de ces pièces syntaxiques, voir la Section III ci-après. 
— En outre l'assignation des macrorôles ACTOR et/ou UNDERGOER ${ }^{6}$ à une ou deux positions argumentales de la structure logique permet de gérer certaines propriétés de la sémantique nominale (caractère référentiel ou non des syntagmes nominaux en position argumentale) ${ }^{7}$ et de la sémantique verbale (diathèse médiopassive en particulier $)^{8}$.

L'articulation des quatre composantes et l'assignation des macrorôles sont représentés dans la Figure 1.

Figure 1 : Les quatre composantes des représentations intégrées

(syntaxiques, logico-sémantiques et pragmatiques) en $R R G$

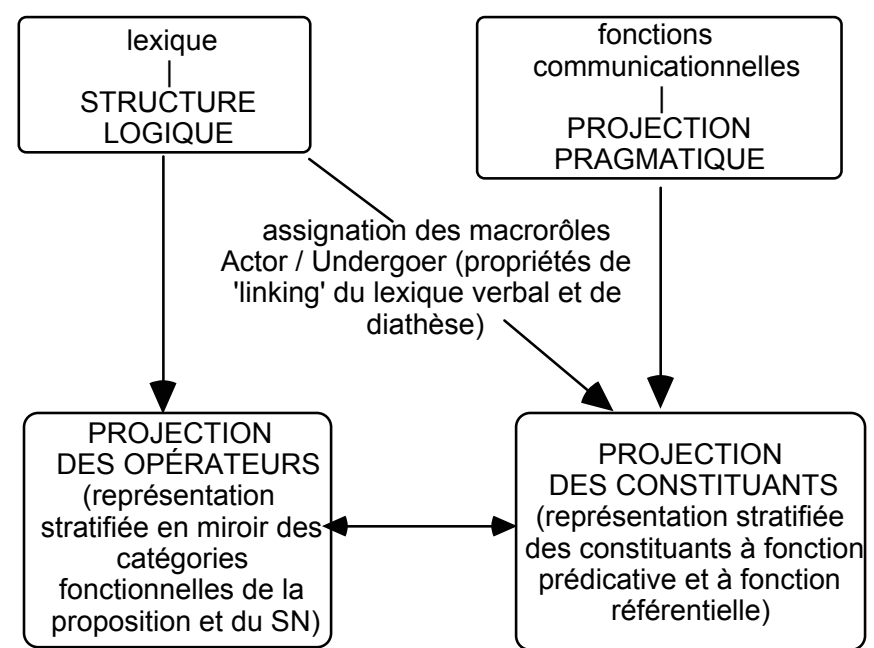

\footnotetext{
${ }^{5}$ Nous considérerons comme équivalents fonction topicale ou thématique et fonction focale ou rhématique.

${ }^{6}$ Le macrorôle ACTOR correspond approximativement au rôle 'prototypique' AGENT et le macrorôle UNDERGOER au rôle prototypique PATIENT de Dowty (1991), mais plus encore aux arguments 1 et 2 de la fonction AFFECT de Jackendoff (1990), laquelle constitue le tiroir actionnel (action tier) en complément du tiroir des rôles thématiques (thematic tier).

${ }^{7}$ Par exemple dans Paul a bu une canette de bière, l'objet est référentiel et reçoit le macrorôle undergoer, tandis que dans Paul a bu \{de la / beaucoup de / un peu de $\}$ bière, l'objet est considéré par R. van Valin comme non référentiel (cf. François à paraittre 2) et ne reçoit aucun macrorôle.

${ }^{8}$ Par exemple, dans Le vent a cassé la branche, le sujet et l'objet reçoivent respectivement les deux macrorôles ACTOR et UNDERGOER, tandis que dans La branche s'est cassée (sous l'effet du vent), un seul macrorôle UNDERGOER peut être assigné au sujet du verbe se-casser, la mention du vent relevant de la 'périphérie'. Dans la Structure Logique, l'entrée lexicale à un seul argument du verbe se-casser est dans la portée d'un prédicat supérieur CAUSE dont le premier argument est en notation informelle $<$ la branche se-cass- $>$ et le second argument $<$ le vent $>$.
} 
Jacques François

\section{La Structure logique}

Tout au long de cet article, nous ne prendrons en compte que le triplet de trois énoncés (1-3) :

(1) Jean a offert des fleurs à Marie.

(2) Jean, des fleurs, il en a offert à Marie.

(3) Qu'est-ce que Jean a offert à Marie?

L'énoncé (1) a une force illocutoire déclarative sans marquage topical ni focal. Le prédicat verbal OFFRIR à la voix active est accompagné d'une instanciation des trois arguments convoqués par son entrée lexicale offrir (x1, x2, à x3); $\mathrm{x} 1$ et $\mathrm{x} 3$ sont instanciés chacun par un nom propre, $\mathrm{x} 2$ par un syntagme nominal indéfini pluriel des fleurs. L'énoncé (2) a le même type de force illocutoire, mais il se caractérise dans la terminologie de van Valin par une dissociation du rôle et de la référence pour x1 et $\mathrm{x} 2$ : le rôle d'agent et la fonction de sujet sont assumés pour $\mathrm{x} 1$ par un pronom clitique masculin $i l$, tandis que le nom propre Jean antéposé (constituant extra-propositionnel) assume la fonction de référence jointe à son statut topical. Pour x2, le rôle d'Affecté et la fonction d'objet direct sont assumés par le pronom partitif en et la référence par le SN extra-propositionnel des fleurs également topical. Cette dissociation entre rôle et référence montre que le français oral présente des caractéristiques des langues à marquage sur la tête (les pronoms clitiques étant assimilés à des affixes verbaux) contrairement au français écrit qui relèverait partiellement du type des langues à marquage sur les membres (par le régime prépositionnel des arguments obliques). Enfin l'énoncé (3) a une force illocutoire interrogative marquée par le morphème est-ce que et l'intonation finale montante et $\mathrm{x} 2$ est introduit comme non référentiel et focal par l'intermédiaire du pronom interrogatif $q u(e)$.

\section{- Structure logique de l'énoncé (1)}

La structure logique de l'énoncé (1) se compose de quatre constituants :

(i) En premier lieu l'entrée lexicale du prédicat verbal offrir, présentée sous une forme décomposée empruntée à Dowty (1979). Cette entrée a un caractère aspectuel $\mathrm{d}^{\prime}$ Accomplishment ${ }^{9}$ et un caractère participatif causatif. L'Accomplishment est noté par la composante BECOME avoir' (x2, x3) qui indique la transition entre 'x2 n'a pas x3' et 'x2 a x3'. Il se combine avec une Activity ${ }^{10}$ notée do' $(\mathrm{x} 1, \varnothing)$ par l'intermédiaire de l'opérateur CAUSE. L'ensemble se lit ainsi : «x1 accomplit un acte non spécifié $(\varnothing)$, lequel cause la transition entre ne pas avoir et avoir entre x 2 et x 3 ».

(ii) x1 est spécifié par ailleurs comme argument du prédicat nominal Jean, x2 comme argument du prédicat nominal Marie et x3 comme une quantité indéfinie de fleurs.

\footnotetext{
${ }^{9}$ Procès doublement caractérisé comme télique et duratif.

${ }^{10}$ Procès atélique et duratif. En principe le type 'Activity' n'implique pas que le référent du sujet contrôle le procès et van Valin \& LaPolla (1997) citent rain et roll (p. 92) et The wheel squeaks (p. 105) comme exemple d'Activities. Cependant la majorité des exemples évoqués concernent des êtres humains contrôlant le procès. De ce fait, l'interprétation du prédicat abstrait do' est sujette à caution.
} 
(iii) l'entrée lexicale du prédicat verbal offrir complétée par l'instanciation des trois arguments reçoit une référence temporelle (tps : PASSÉ).

(iv) Enfin le tout est affecté d'une force illocutoire déclarative (fi : DECL)

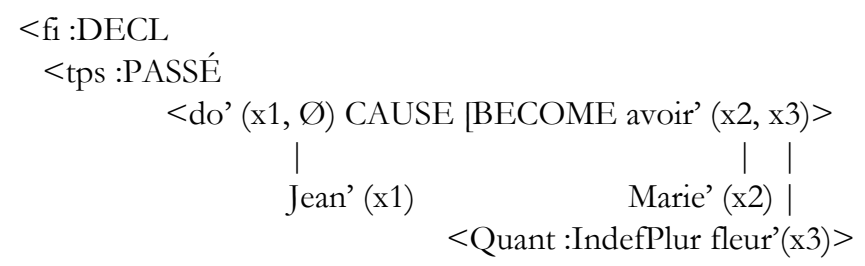

\section{- Structure logique de l'énoncé (2)}

Les énoncés (1) et (2) ont une même structure logique. En (2), la dissociation entre référence et rôle notée plus haut ne concerne pas la structure logique, mais la projection pragmatique par l'intermédiaire de l'association entre fonction référentielle et fonction topicale d'une part et fonction actancielle et statut de clitique de l'autre (Jean...il; des fleurs ... en). Par commodité, je considérerai provisoirement que les fonctions de la projection pragmatique Topic et Focus sont attachées aux symboles référentiels de la structure logique. Dans la présentation par van Valin \& LaPolla (1997) de l'articulation entre les composantes de la représentation intégrée, les fonctions de Topic et Focus contribuent en fait à sélectionner les pièces syntaxiques de la projection des constituants et ne sont pas rattachées directement à la structure logique.

$<$ fi :DECL

$<$ tps :PASSÉ

$<$ do' $(\mathrm{x} 1, \varnothing)$ CAUSE [BECOME avoir' (x2, x3)>

Jean' (x1)

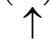

Proj. pragm. : Topic 1

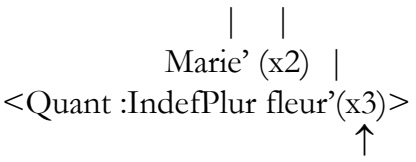

Proj. pragm. : Topic 2

\section{- Structure logique de l'énoncé (3)}

L'énoncé (3) a une structure logique différente des deux énoncés précédents à deux titres: la force illocutoire interrogative et la focalisation de la question sur l'identification de x3. C'est cette double spécification qui 'instruit' la projection des constituants, c'est-à-dire sélectionne les pièces (templates) appropriées. 
Jacques François

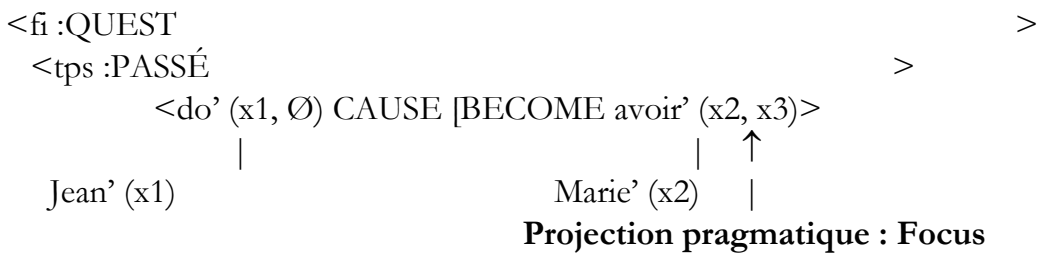

\section{La sélection des pièces de la projection des constituants à partir de la structure logique et de la projection pragmatique}

En l'absence d'instruction en provenance de la projection pragmatique, la structure logique de l'énoncé (1) sélectionne un Cœur ${ }^{11}$ trivalent avec un objet direct et un objet oblique (pièce $\mathrm{n}^{\circ} 1$ ). L'argument 'sujet' est associé à x1 comme premier argument de la fonction do' $(\sim, \varnothing)$ par l'intermédiaire du macrorôle Acteur. L'argument 'objet' est associé à x 3 comme second argument de la fonction BECOME avoir' (x2, ) et l'argument oblique est associé à x2 comme premier argument de la même fonction ${ }^{12}$.

(1) Jean a offert des fleurs à Marie.

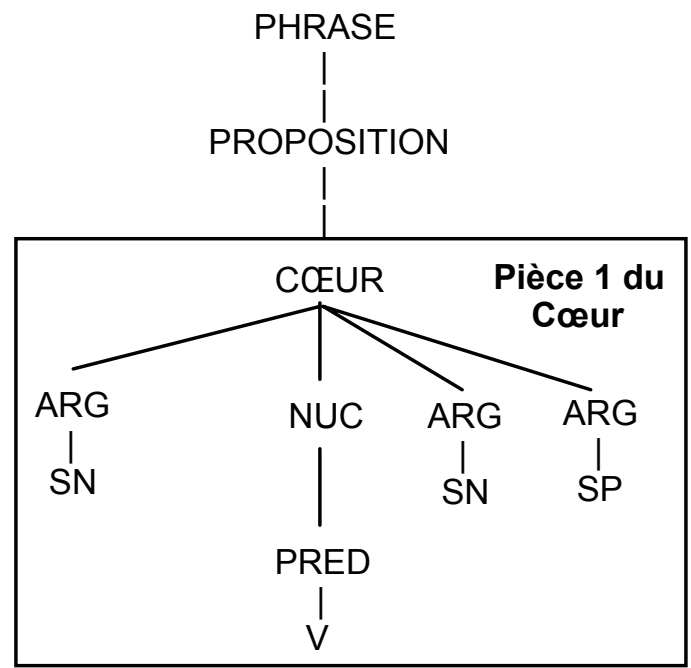

\footnotetext{
${ }^{11}$ La notion de «Cœur propositionnel » en RRG équivaut à celle de "grille thématique » ou de «structure argumentale » dans la théorie du gouvernement et du liage (cf. Grimshaw 1990) et à celle de prédication nucléaire (core predication) dans le modèle néerlandais de grammaire fonctionnelle (cf. Dik 1989).

12 Le nœud Nuc(leus) occupe une position intermédiaire entre le Cœur et le prédicat verbal dans la structure stratifiée de la proposition, destinée à permettre le rattachement d'adverbes de nucleus (par ex. les adverbes de degré) et l'opération de jonction de nuclei (nuclear juncture) par ex. pour traiter la diathèse causative (cf. Paul fait offrir des fleurs à Marie).
} 
L'énoncé (2) comprend en revanche deux constituants topicaux porteurs de référence, mais extra-propositionnels et représentés dans la proposition par deux pronoms clitiques. Les constituants topicaux sont rattachés à deux nœuds désignés comme "position détachée gauche » et dominés par le nœud PHRASE. Il est essentiel de noter que l'assignation d'une position détachée ne résulte pas d'un mouvement à partir de la structure de constituants de l'énoncé $(1)^{13}$. L'instanciation de ces positions est pilotée par la projection pragmatique qui spécifie les constituants topicaux et focaux. La pièce 2 du Cœur se distingue de la pièce 1 par la disposition (à nouveau en aucun cas le résultat d'un mouvement !) du pronom clitique objet à gauche du prédicat verbal.

(2) Jean, des fleurs, il en a offert à Marie.

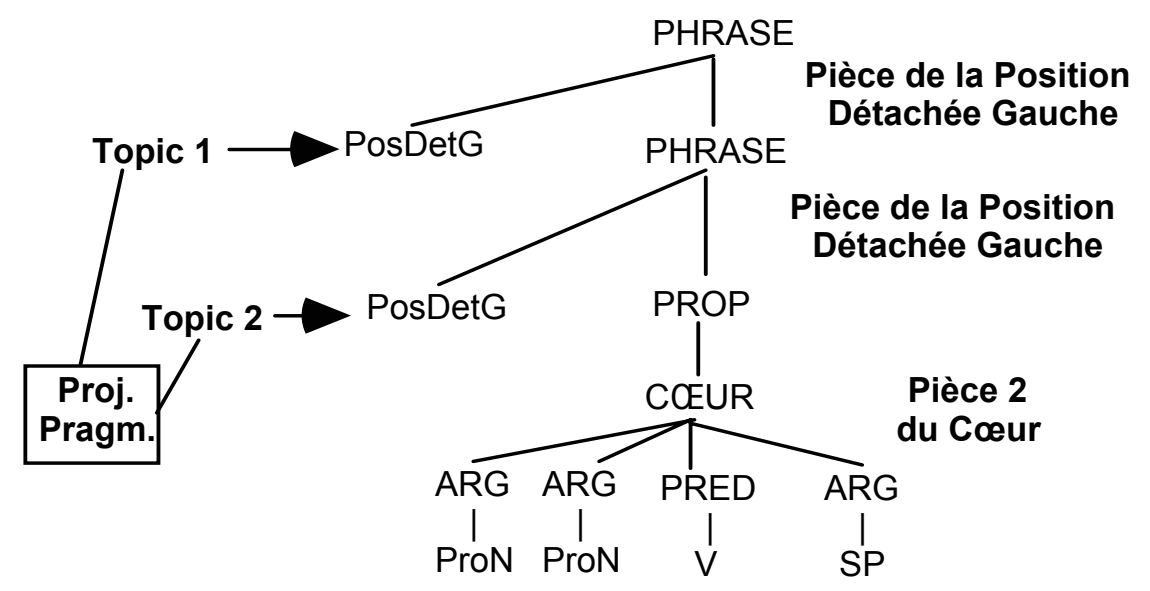

Enfin la structure de constituants de l'énoncé (3) est caractérisée par l'instanciation d'un «créneau pré-cœur» (pre-core slot) destiné à recevoir le pronom interrogatif objet. Cette instanciation est pilotée conjointement par une propriété de la structure logique, la force illocutoire interrogative et une propriété de la projection pragmatique, l'assignation à l'objet du statut de focus (interrogatif). La conséquence de la génération d'un pronom interrogatif objet et de sa disposition dans le créneau précœur est la sélection d'une troisième pièce pour le Cœur, constituée d'un argument 'sujet' pronominal et d'un argument oblique. Encore une fois, cette pièce syntaxique ne comporte pas de «trace» d'un mouvement de l'objet, parce que l'objet n'a pas été déplacé, il a reçu une affectation pilotée par le double jeu de la structure logique et de la projection pragmatique.

\footnotetext{
${ }^{13}$ Cela constitue une différence essentielle avec la théorie du Gouvernement et du Liage. Malgré la terminologie employée, il n'y a pas à proprement parler de «détachement ", mais plutôt comme le formule bien Dik (1997, Chapter 17: Extraclausal constituents) la sélection par la projection pragmatique d'une position «extrapropositionnelle» (cf. Lacheret-Dujour \& François, à paraître). Aucune structure n'est à la source d'un mouvement dont une autre structure serait la cible et dont la structure source garderait la trace.
} 
Jacques François

(3) Qu'est-ce que Jean a offert à Marie?

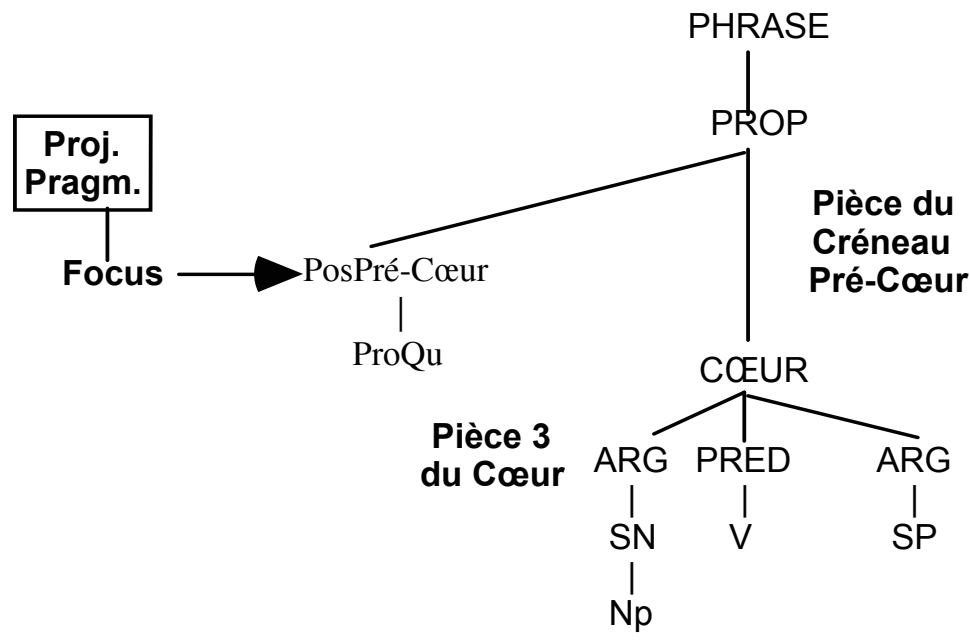

\section{L'intégration entre les données des quatre composantes}

J'ai évoqué pour la sélection des pièces de la Phrase, de la Proposition et du Cœur la source de cette sélection (structure logique, assignation des macrorôles Acteur et Patient, assignation des fonctions topicale et focale). Il reste encore à évoquer un caractère fondamental des représentations syntaxiques de la RRG, la distribution du matériau morphosyntaxique entre la projection des constituants et la projection des opérateurs du constituant prédicatif (le verbe) et de chacun des constituants référentiels (les $\mathrm{SN}$ ).

L'énoncé (1) est au passé (composé) et a une force illocutoire déclarative. Ces deux propriétés sont spécifiées dans la structure logique sous la forme de prédicats superordonnés à l'entrée lexicale du prédicat verbal et sont associées à des strates de la projection des opérateurs de la proposition. L'instanciation PASSÉ de l'opérateur de temporalité de la strate Proposition sélectionne le passé composé ${ }^{14}$ cependant que la force illocutoire déclarative est par convention associée à ce même auxiliaire. Par ailleurs, sur la projection des opérateurs du N fleur, l'opérateur de quantification est instancié par la valeur de déterminant indéfini pluriel. Ainsi les constituants $a$, forme fléchie de l'auxiliaire de temps avoir et des, forme fléchie de l'article indéfini, sont

\footnotetext{
${ }^{14} \mathrm{La}$ sélection distinctive des temps du passé (passé composé vs. simple vs. imparfait) demanderait un traitement plus sophistiqué que ce qui est actuellement proposé, par exemple en assignant à chaque emploi d'un tiroir verbal une valeur de l'opérateur de temps et une valeur de l'opérateur de visée aspectuelle, comme l'ont proposé Combettes, François, Noyau \& Vet (1993) dans le prolongement de la logique temporelle de H. Reichenbach.
} 
sélectionnés par la projection des opérateurs et en quelque sorte «mis à la disposition» de la projection des constituants. Ils sont considérés comme des opérateurs par leur position aussi bien dans la structure logique que dans la projection des opérateurs ${ }^{15}$.

(1) Jean a offert des fleurs à Marie.

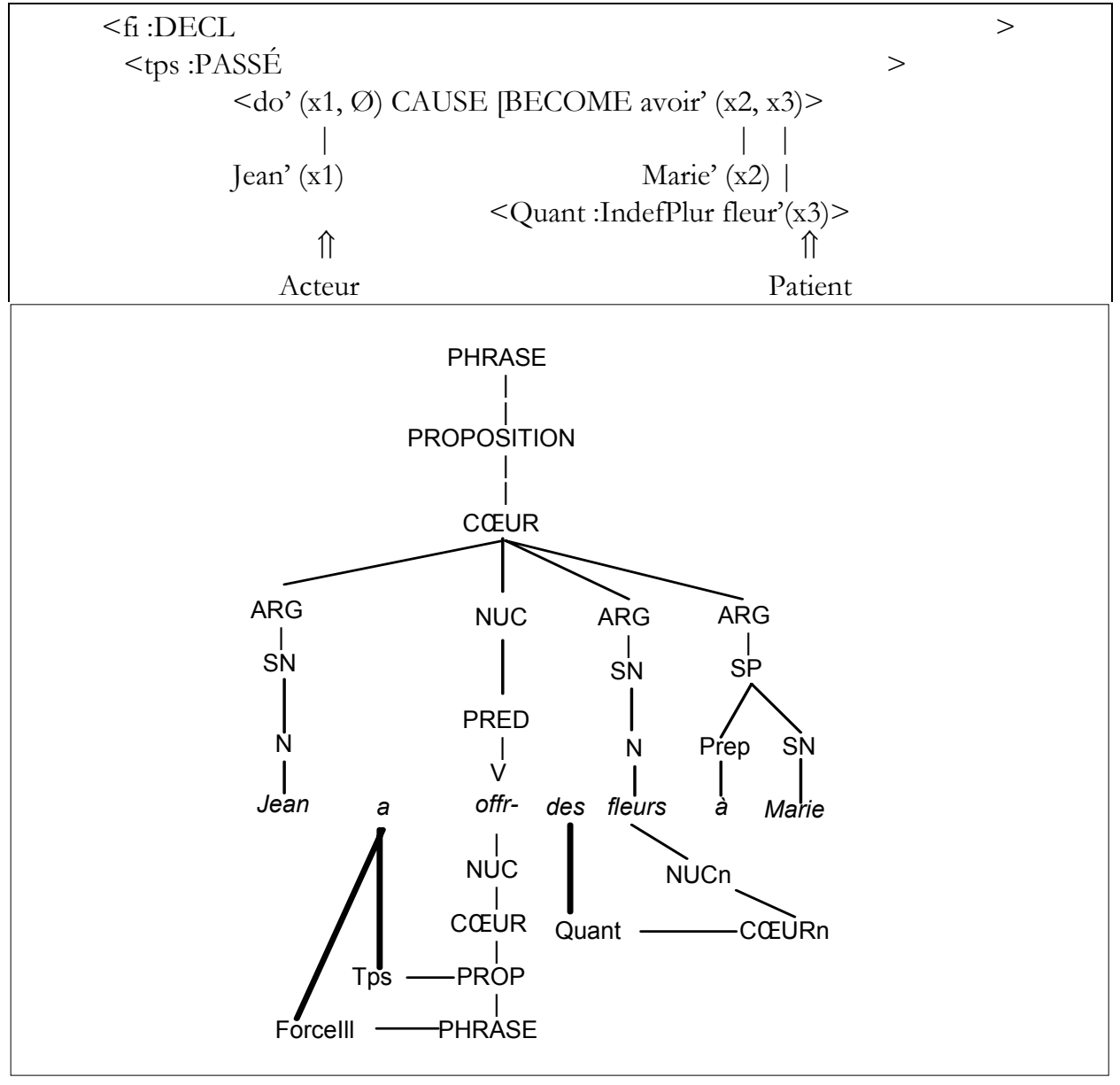

Pour l'énoncé (2), la projection des opérateurs de la proposition et celle du SN des fleurs sont identiques à celles de l'énoncé (1). Seule la disposition extrapropositionnelle du SN objet diffère.

${ }^{15}$ C'est tellement vrai qu'on peut presque parler de «variante notationnelle» entre les deux composantes et qu'on pourrait envisager une notation compacte intégrant la partie supérieure de la structure logique et la projection des opérateurs. 
(2) Jean, des fleurs, il en a offert à Marie.

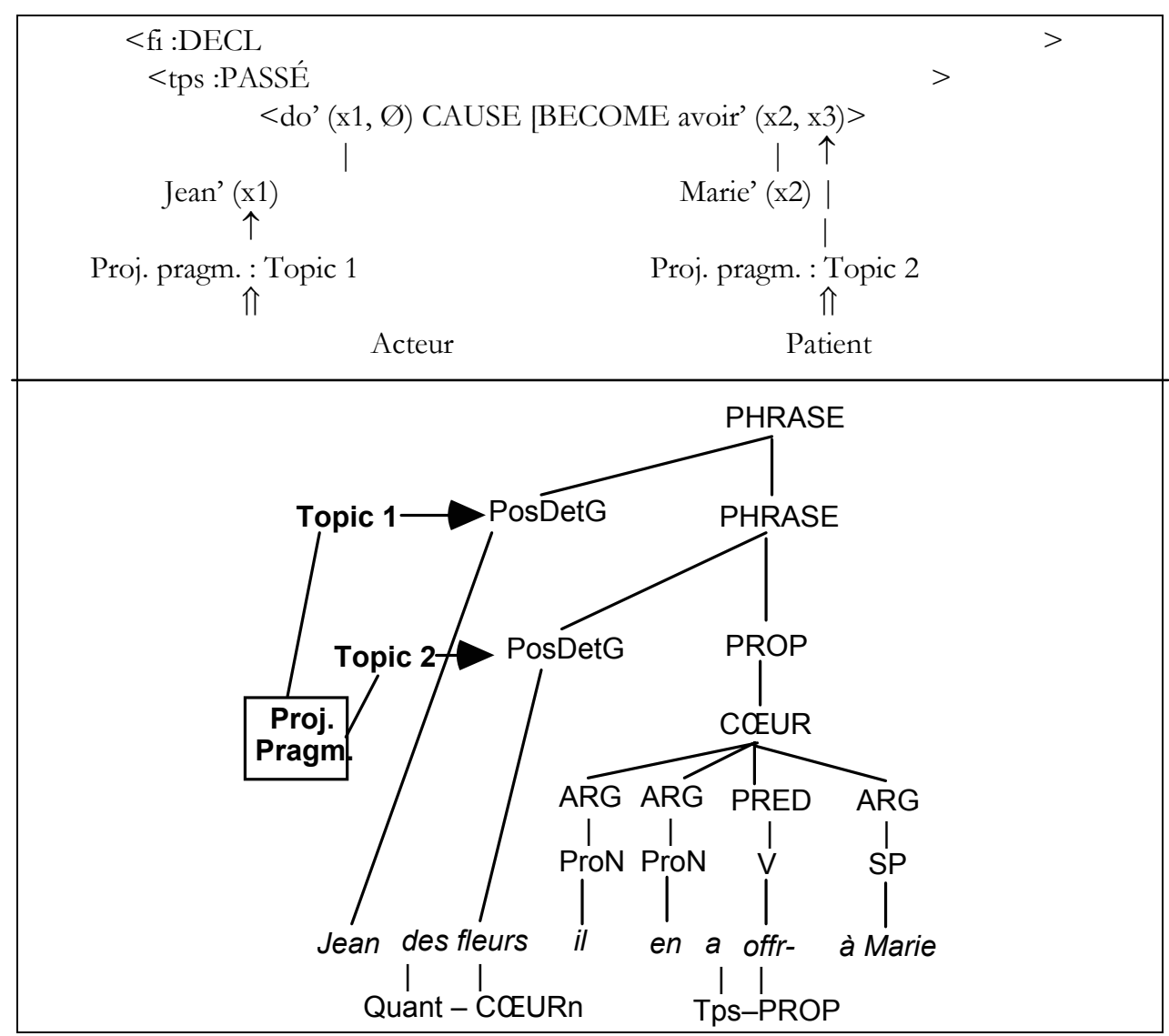

Pour l'énoncé interrogatif (3), le pronom interrogatif que est dénué de projection des opérateurs et le morphème est-ce que est le marqueur de la force illocutoire interrogative.

(3) Qu'est-ce que Jean a offert à Marie ?

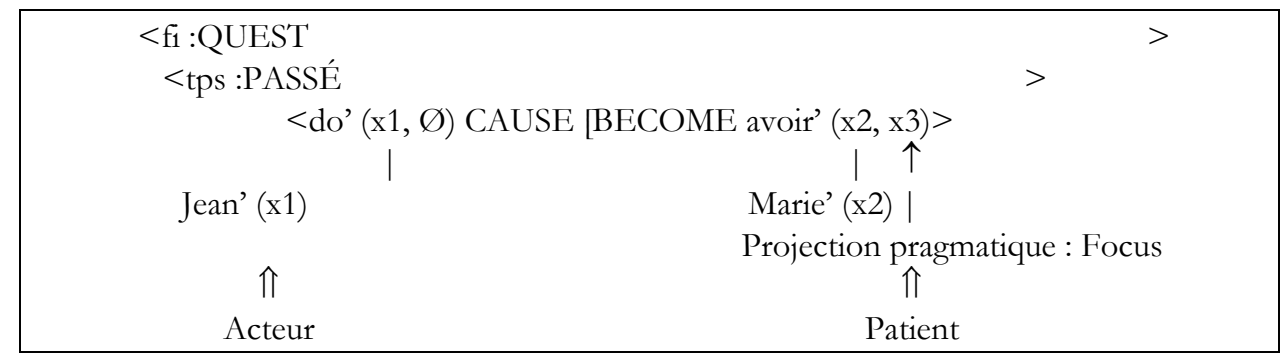




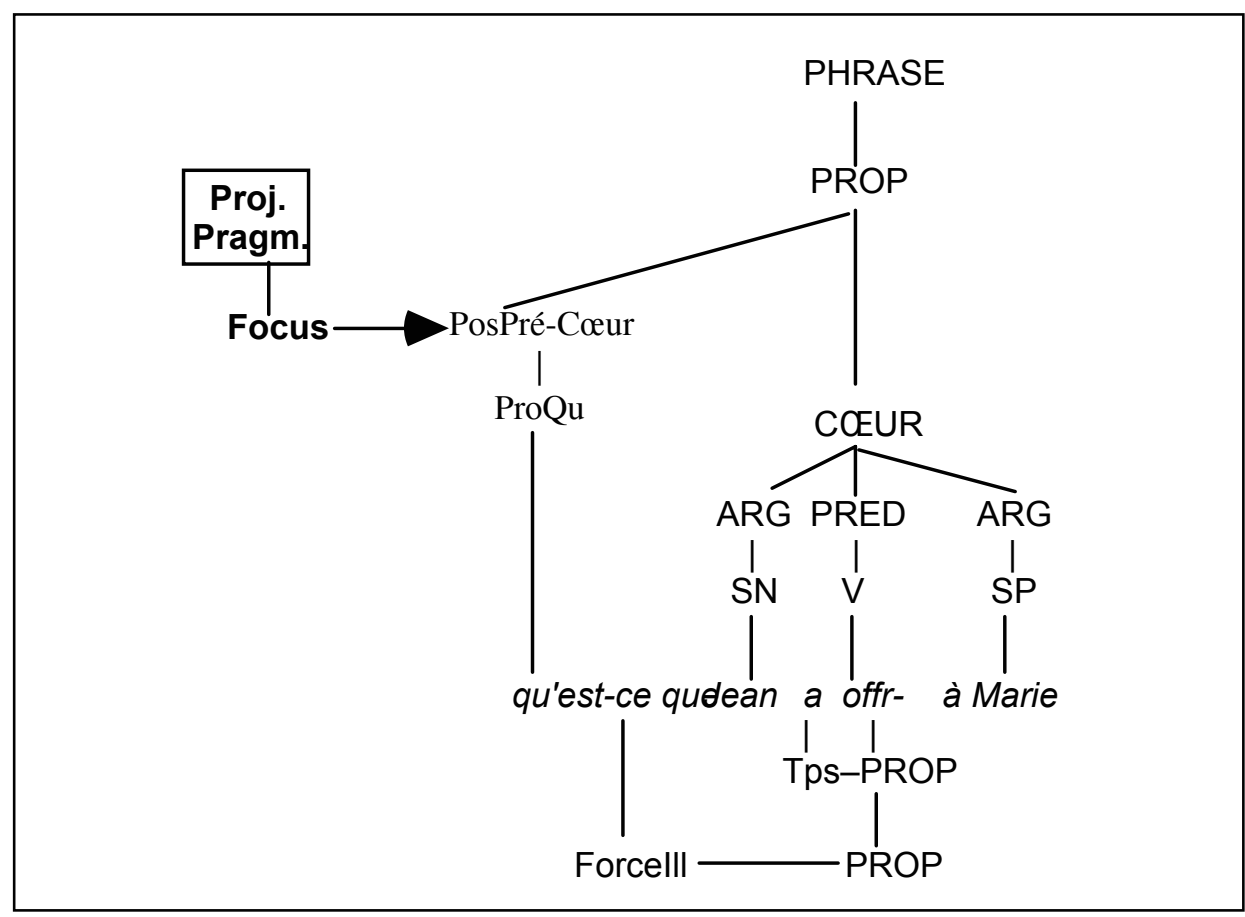

\section{Une grammaire "distribuée » et dénuée de «mouvements" de constituants}

Pour conclure, je voudrais souligner les deux caractéristiques distinctives majeures entre la RRG et les syntaxes «dérivationnelles » (modèles du gouvernement et du liage, programme minimaliste).

(I) La RRG, de même que le modèle néerlandais de grammaire fonctionnelle de Dik $(1989,1997)$, mais avec une composante syntaxique plus explicite et maniable, ne prévoit aucune transformation et singulièrement aucun mouvement de constituants, parce que ceux-ci sont disposés dans la projection des constituants en fonction d'instructions délivrées par la structure logique (en particulier pour les diathèses) et par la projection pragmatique (pour l'expression de la force illocutoire et la sélection des constituants topicaux et focaux).

(II) D'autre part, pour ce qui concerne l'articulation entre la projection des constituants consacrée en priorité aux constituants à fonction prédicative (le prédicat verbal en tant que tête du Cœur propositionnel) et à fonction référentielle (les têtes nominales) et son image miroir, les projections fonctionnelles destinées à prendre en charge les opérateurs propositionnels (aspect, temps, modalité, expression grammaticale de la force illocutoire) et les opérateurs de la référence nominale (détermination, qualification, quantification), van Valin \& LaPolla mettent en évidence les similarités superficielles et les divergences profondes entre les deux approches (je cite partiellement van Valin \& LaPolla 1997 : 652, note 20) : 
Jacques François

- Les similarités entre les théories $G B$ et $R R G$ sur la conception de la projection fonctionnelle (ou projection des opérateurs)

- Un SV contenant le sujet en GB correspond grossièrement au 'Cœur' en RRG.

- Les projections fonctionnelles supérieures en GB correspondent grossièrement à la projection des opérateurs en RRG.

- Les trois différences de fond entre les deux conceptions

\section{GB}

Les morphèmes d'accord verbal sont inclus parmi les projections fonctionnelles. Les $\mathrm{SN}$ et le verbe se déplacent de leurs positions initiales internes au SV le long de l'arbre pour prendre le cas, vérifier l'accord, etc.

Il n'y a pas de motivation de principe ${ }^{16}$ pour la disposition hiérarchique des catégories fonctionnelles; c'est-à-dire qu'il n'y a pas d'explication de principe de la raison pour laquelle le syntagme temporel devrait être plus haut dans l'arbre que le syntagme aspectuel.

\section{RRG}

Les morphèmes d'accord ne sont pas considérés comme des opérateurs.

Les éléments de la projection des constituants n'interagissent avec les éléments de la projection des opérateurs que du point de vue de la linéarisation. L'accord et l'assignement du cas sont traités dans la corrélation entre la représentation sémantique [i.e. la structure logique] et la projection de constituants; la projection des opérateurs ne joue aucun rôle direct dans la prise en compte de ces phénomènes.

Il y a une explication sémantique claire pour la disposition hiérarchique des opérateurs dans la projection des opérateurs.

Il reste bien entendu des problèmes de représentation à résoudre, par exemple le statut différencié des adverbes, qui sont traités en RRG dans la projection des constituants, et des adjectifs 'qualificatifs' qui relèvent de la projection des opérateurs. Ce rattachement empêche de leur attribuer une projection des opérateurs propre, dont aucun syntagme adjectival en fonction déterminative ne peut se passer. Cependant, entre les deux extrêmes des modèles de syntaxe 'dérivationnelle' centrés sur une syntaxe parfaitement autonome, et du modèle néerlandais de grammaire fonctionnelle

\footnotetext{
${ }^{16}$ Van Valin \& LaPolla entendent par là que la hiérarchisation des catégories fonctionnelles n'est pas motivée par des considérations de portée fonctionnelle respective (ainsi l'expression de la visée aspectuelle prend fonctionnellement dans sa portée l'expression de la phase et non l'inverse, cf. $A 8 \mathrm{~h}$, Marie était en train de commencer à dîner et non * A 8 b Marie commençait à être en train de dîner).
} 
centré sur la représentation polyvalente (sémantique, pragmatique et catégorielle) d'une «structure sous-jacente » articulée sur des règles d'expression morphologique, de linéarisation et de contour prosodique où la syntaxe n'a aucune autonomie, la RRG constitue actuellement un compromis équilibré qui préserve une composante syntaxique maniable, mais en quelque sorte «asservie » aux besoins de la sémantique et de la pragmatique. Le titre de l'ouvrage à paraître de van Valin The Syntax-SemanticsPragmatics Interface : an Introduction to Role and Reference Grammar est donc parfaitement justifié.

\section{Jacques FRANÇOIS}

\section{RÉFÉRENCES}

COMBETTES, B., J. FRANÇOIS, C. NOYAU \& C. VET (1993), Introduction à l'étude des aspects dans le discours narratif. Verbum 1993/4, 5-48.

DIK, S.C. (1989), The Theory of Functional Grammar, Part 1 : The Structure of the Clause. Dordrecht: Foris.

DIK, S.C. (1997), The Theory of Functional Grammar, Part 2 : Complex and derived constructions. Dordrecht : Foris.

DOWTY, D. (1979), Word Meaning and Montague Grammar. Dordrecht : Reidel.

FRANÇOIS, J. (à paraître 1), La représentation des structures verbo-nominales et du figement verbal dans deux formalismes de grammaire fonctionnelle. Cabiers de lexicologie 82, 2003.

FRANÇOIS, J. (à paraitre 2), Types de prédications et statut participatif et référentiel de l'objet : l'approche de la Role and Reference Grammar. In: F. Corblin et al. Actes du Colloque Indéfinis et prédication. Université de Paris-IV-Sorbonne, octobre 2002. Presses de la Sorbonne.

GOLDSMITH, J. \& G. HUCK (1991). Distribution et médiation dans le développement de la théorie linguistique. Communications 53 :51-67.

GRIMSHAW, J. (1991), Argument structure. Cambridge (Mass.) : M.I.T. Press.

JACKENDOFF, R. (1990), Semantic structures. Cambridge (Mass.) : M.I.T. Press.

LACHERET-DUJOUR, A. \& J. FRANÇOIS (à paraitre), De la notion de détachement topical à celle de constituant thématique extrapropositionnel. Cabiers de Praxématique $\left[\mathrm{n}^{\circ}\right.$ sur les constructions détachées dirigé par F. Neveu].

VAN VALIN Jr., R.D. (2001) Introduction to syntax. Cambridge : Cambridge University Press.

VAN VALIN Jr., R.D. (à paraittre), The Syntax-Semantics-Pragmatics Interface : an Introduction to Role and Reference Grammar. Cambridge : Cambridge University Press.

VAN VALIN Jr., R.D. \& R. LaPolla (1997), Syntax : Structure, Meaning, Function. Cambridge: C.U.P. 